\title{
Mycobacterial immunotherapy for prostate cancer: where can we go from here?
}

\author{
Vasilis Stavrinides $^{1,2 \star}$, Angus Dalgleish ${ }^{3}$, John P. Copier ${ }^{3}$ and Caroline M. Moore ${ }^{1,2}$ \\ ${ }^{1}$ Division of Surgery and Interventional Science, University College London, London, UK. \\ ${ }^{2}$ Department of Urology, University College London Hospital NHS Foundation Trust, London, UK. \\ ${ }^{3}$ Oncology Group, Institute of Infection and Immunity, St George's University of London, London, UK. \\ *e-mail:v.stavrinides@ucl.ac.uk
}

The effects of mycobacteria in prostate cancer have not been fully elucidated. Results of early studies indicated that mycobacterial immunotherapy conferred a survival benefit in patients with advanced prostate cancer, and recent evidence supports the safety and efficacy of new mycobacterial agents in many malignancies; thus, modern, carefully designed, randomized controlled trials of mycobacterial immunotherapy for prostate cancer could be warranted.

Impressive advances in the field of prostate cancer immunotherapy have been made in the past decade. For example, sipuleucel-T was approved by the FDA in April 2010 for use in patients with metastatic castration-resistant prostate cancer. However, the clinical results remain modest: overall survival is prolonged by only a few months and no benefit is seen in progression-free survival; furthermore, checkpoint inhibition suffers from similar limitations ${ }^{1}$. Thus, the success of immunotherapies in the context of genitourinary malignancies remains confined to the use of BCG for non-muscle-invasive bladder carcinomas. Even so, our understanding of molecular immunology has increased substantially in recent years, and new ways of expanding the role of immunotherapy are constantly being considered.

The development of prostate cancer immunotherapies is inherently complex. Finding the optimal scheme for administration and using wellvalidated biomarkers or volumetric criteria of response are considerable challenges in clinical trial design. Also, prostatic malignancies have biological characteristics widely considered to be unfavourable for immunotherapy, such as a low immune cell density or mutational burden. However, high infiltrating immune cell density or mutational burdens do not necessarily correlate with clinical benefit ${ }^{2}$. Also, the introduction of PSA screening has resulted in a large diagnostic migration towards low-risk tumours that need to be addressed but not overtreated. Interestingly, patients with low disease burden are less likely to have tumour-induced immune dysfunction and, therefore, could be better suited for immunotherapies that generate early antitumour responses. Ideally, such therapies should be safe to administer in patients with low-stage prostate cancer, dampen cancer-induced immunosuppression, recruit appropriate populations of effector cells in the tumour microenvironment, induce measurable effects in tissues or biological fluids and be easily combined with existing treatments without increasing toxicity risk.

Some evidence suggests that mycobacterial preparations could fulfil one or more of these criteria. Work on animal and cell line models has previously shown that mycobacterial extracts inhibit the proliferation of transformed cells and restore $\mathrm{T}$ helper 1 ( $\left.\mathrm{T}_{\mathrm{H}} 1\right)$ cell immunity, while recently discovered mycobacterial strains, such as Mycobacterium vaccae and Mycobacterium obuense, are able to stimulate $\gamma \delta$ T cells, a critical component of antitumour immunity ${ }^{3}$. Whether these results can be translated to treatment of patients with prostate cancer is currently unknown, but some lessons can be learned from the administration of mycobacteria against various malignancies. For example, SRL172 (heat-killed M. vaccae) has been administered to patients with metastatic renal cell carcinoma (RCC) after nephrectomy ${ }^{4}$. No statistically significant differences in survival were seen between the SRL172 and the biological therapy (IL-2 or TNF) arm, but the survival of patients receiving chemotherapy was worse than that of patients who received other treatments $(P<0.001)$, suggesting that SRL172 is at least as effective as traditional disease modifiers. Another group randomized 419 patients with stage III-IV non-small-cell lung cancer to SRL172 plus chemotherapy versus chemotherapy alone. No difference in overall survival was initially found, but re-analysis of the study data (based on the observation that only $53 \%$ of patients in the combination arm received more than two SRL172 injections) indicated that patients with non-squamous lung adenocarcinoma adhering to the full dosing schedule lived on average 357 days (95\% CI 293-421) versus only 222 days (95\% CI 180-263) in the chemotherapy group ${ }^{5}$. To date, only a few formal trials have investigated the effects of intradermal IMM-101 (heat-killed M. obuense) immunotherapy; the largest was a phase II study (NCT01303172) assessing the safety and efficacy of IMM-101 combined with gemcitabine in 110 patients with locally advanced or metastatic pancreatic adenocarcinoma ${ }^{6}$. Overall survival for the intention-to-treat population was 6.7 months for IMM-10 plus gemcitabine versus 5.6 months for gemcitabine alone (HR 0.68, 95\% CI 0.44-1.04, $P=0.074$ ). Differences in overall survival between the two arms were also observed in the metastatic disease subgroup (OS 7.0 versus 4.4 months; HR 0.54, 95\% CI 0.33-0.87, $P=0.01$ ), whereas injection site reactions were mild to moderate.

With regard to prostate cancer, most studies on mycobacterial immunotherapies were performed before the study of molecular biology or monoclonal antibody technology and are, therefore, difficult to interpret. However, in a 1973 trial that aimed to determine the effect of BCG on survival and immunological parameters in patients with prostate cancer of all stages, interim reports showed a favourable safety profile of intradermal administration. Overall survival differences between the intervention and control arms reached statistical significance (OS for all stages: 37.5 versus 21 months, $P=0.02$ ), whereas stage-specific survival differences were larger in lower stage disease. Although initially all patients were treated, stratification and randomization were consistent after 1976, and the results indicated that intradermal BCG is effective, safe and able to stimulate the immune system. In a 1998 phase I/II trial, SRL172 was administered in ten patients with hormone-resistant, advanced prostate cancer $^{8}$. The injection only caused mild redness and swelling $(5-8 \mathrm{~mm})$ at the administration site in most patients and fever in one, but no other major systemic reactions were recorded. PSA reduction was seen in five men, two of whom received SRL172 as their only treatment. The results also indicated that men with advanced prostate cancer have a $\mathrm{T}_{\mathrm{H}} 2$ cytokine profile within their peripheral blood mononuclear cells, which predominantly secrete IL-4. Interestingly, SRL172 vaccination was associated with IL-2 production in three of ten men, a cytokine considered to be a crucial component of cell-mediated immunity.

Collectively, these results indicate that mycobacteria are safe, well tolerated and could shift immune responses from $\mathrm{T}_{\mathrm{H}} 2$ (predominantly humoral and associated with cancer progression) to $\mathrm{T}_{\mathrm{H}} 1$ (predominantly cell-mediated and associated with antitumour responses). Improvements in trial design could enable the study of mycobacterial strains that are well tolerated when delivered systemically (such as M. vaccae and 
M. obuense) and could, therefore, be administered early in the treatment pathway, before prostate cancer-induced or treatment-related immune dysfunction is established. Experimental evidence supports the notion that early disease modulation could be effective; for example, the therapeutic vaccination of TRAMP mice against specific prostate tumour antigens is more effective in mice with prostatic intraepithelial neoplasia than those with already established cancers ${ }^{9}$. Monitoring tumour responses in early stages is challenging, but recent advances in imaging could enable the longitudinal monitoring of low-risk tumours and facilitate repeated sampling of a particular disease focus. Other interventions have been previously tested in this manner ${ }^{10}$ and it could be possible to extend these methodologies in immunotherapy trials. Whether trials like these would have any practical value remains to be seen, but in an era in which effective prostate cancer treatments are highly desirable, having a fresh, critical look into concepts previously dismissed as, apparently, ineffective could be a way forward.

1. Boettcher, A. N. et al. Past, current, and future of immunotherapies for prostate cancer. Front. Oncol. https://doi.org/10.3389/fonc.2019.00884 (2019).

2. Abida, W. et al. Analysis of the prevalence of microsatellite instability in prostate cancer and response to immune checkpoint blockade. JAMA Oncol. 5, 471-478 (2018).

3. Bazzi, S. et al. Analysis of the immunomodulatory properties of two heat-killed mycobacterial preparations in a human whole blood model. Immunobiology 220, 1293-1304 (2015).

4. Patel, P. M. et al. An evaluation of a preparation of Mycobacterium vaccae (SRL172) as an immunotherapeutic agent in renal cancer. Eur. J. Cancer 44, 216-223 (2008).

5. Stanford, J. L. et al. Successful immunotherapy with Mycobacterium vaccae in the treatment of adenocarcinoma of the lung. Eur. J. Cancer 44, 224-227 (2008).

6. Dalgleish, A. G. et al. Randomised, open-label, phase II study of gemcitabine with and without IMM-101 for advanced pancreatic cancer. Br. J. Cancer 115, 789-796 (2016).

7. Guinan, P. et al. Adjuvant immunotherapy with bacillus Calmette-Guérin in prostatic cancer. Urology 14, 561-565 (1979).

8. Hrouda, D. et al. Immunotherapy of advanced prostate cancer: a phase I/II trial using Mycobacterium vaccae (SRL172). Br. J. Urol. 82, 568-573 (1998).

9. Gray, A. et al. Prostate cancer immunotherapy yields superior long-term survival in TRAMP mice when administered at an early stage of carcinogenesis prior to the establishment of tumorassociated immunosuppression at later stages. Vaccine 27, G52-G59 (2009).

10. Moore, C. M. et al. The effect of dutasteride on magnetic resonance imaging defined prostate cancer: mapped-a randomized, placebo controlled, double-blind clinical trial. J. Urol. 197, 1006$1013(2017)$.

Acknowledgements

The authors thank the Institute for Cancer Vaccines and Immunotherapy (project code 517932, award number 164271) for support. V.S. is supported by a Medical Research Council (MRC) Clinical Research Training Fellowship (MR/S005897/1), a European Association for Cancer Research Travel Fellowship and a University College London Bogue Fellowship. C.M.M. receives funding from Movember, Prostate Cancer UK, the National Institute for Health Research, the European Association of Urology Research Foundation and the MRC.

Competing interests

The authors declare no competing interests.

Pullquote

mycobacteria are safe, well tolerated and could shift immune responses 